\title{
The Relationship Between Existence of Typical Symptoms and Psychological Factors in Patients With Erosive Esophagitis
}

Sang Pyo Lee, Kang Nyeong Lee, Oh Young Lee, ${ }^{*}$ Hang Lak Lee, Ho Soon Choi, Byung Chul Yoon, Dae Won Jun, Won Sohn and Seung Chul Cho

Department of Internal Medicine, Hanyang University College of Medicine, Seoul, Korea

\section{Background/Aims}

In Asian countries including Korea, the prevalence of gastroesophageal reflux disease (GERD) is on the rise and its clinical impact has been emphasized. The purpose of this study was to investigate the clinical characteristics of esophagitis patients with or without symptoms, and their association with psychological factors.

\section{Methods}

Subjects diagnosed as erosive esophagitis of Los Angeles-A or more in screening by upper gastrointestinal endoscopy were enrolled. Questionnaires regarding GERD symptoms and Symptom Checklist-90-Revision were used to identify the presence of psychological symptoms.

Results

There was no difference between the subjects' general characteristics (gender, age, body mass index, smoking and alcohol intake) according to the existence of typical symptoms in these patients with erosive esophagitis. Patients with typical GERD symptoms were more likely to have atypical symptoms, dyspepsia and higher scores on psychological symptoms (somatization, obsessive-compulsiveness and phobic anxiety) than those without.

\section{Conclusions}

Psychological symptoms and other gastrointestinal symptoms should be considered in the patients with erosive esophagitis. (J Neurogastroenterol Motil 2012;18:284-290)

\section{Key Words}

Eosphagitis; Gastroesophageal reflux; Psychological test

Received: November 16, 2011 Revised: February 20, 2012 Accepted: February 26, 2012

(c) This is an Open Access article distributed under the terms of the Creative Commons Attribution Non-Commercial License (http://creativecommons. org/licenses/by-nc/3.0) which permits unrestricted non-commercial use, distribution, and reproduction in any medium, provided the original work is properly cited.

*Correspondence: Oh Young Lee, MD, PhD

Department of Internal Medicine, Hanyang University College of Medicine, 222 Wangsimni-ro, Seongdong-gu, Seoul 133-792, Korea

Tel: +82-2-2290-8343, Fax: +82-2-2298-9183, E-mail: leeoy@hanyang.ac.kr

Financial support: None.

Conflicts of interest: None. 


\section{Introduction}

Gastroesophageal reflux disease (GERD) is defined as a disorder in which gastric contents reflux recurrently into the esophagus, causing troublesome symptoms and/or complications. ${ }^{1}$ This disorder can be classified into non-erosive reflux disease (NERD), erosive relux disease (ERD), Barrett's esophagus and so on, depending on the endoscopic findings. ${ }^{2}$

In the West, the proportion of the people experiencing typical GERD symptoms such as heartburn and acid regurgitation reaches $20 \%-40 \%$ in the general population and the prevalence of GERD is known to be $10 \%-20 \% .^{3-6}$ In Korea, the prevalence of patients with typical GERD symptoms is as low as 3.5\%$8.5 \% .^{7-13}$ In Korea and other Asian countries, the prevalence of GERD is lower than in the West but, has been increasing and its importance has recently been emphasized. ${ }^{1,14}$

GERD symptoms may develop in response to psychosocial factors as well as organic etiologies. ${ }^{15,16}$ Psychosocial factors can affect the development of symptoms, responses to treatment and quality of life. ${ }^{16,17}$ There have been many studies on the relationship between GERD symptoms and psychosocial factors. ${ }^{15,16,18,19}$ However, few studies restricted enrollment of patients with endoscopically-confirmed erosive esophagitis. In addition, not much is known about the differences in characteristics between symptomatic and asymptomatic GERD patients. ${ }^{20}$

In the present study, we compared the baseline characteristics of patients with or without typical GERD symptoms. We investigated whether atypical symptoms of GERD and symptoms of functional dyspepsia (FD) are associated with the presence of typical symptoms of GERD. And we also investigated the correlation of typical GERD symptoms with psychological factors in esophagitis patients.

\section{Materials and Methods}

From September 2007 to September 2008, among the patients who visited the Hanyang University Hospital for a routine check-up, patients diagnosed with erosive esophagitis Los Angeles (LA)-A or more were enrolled. At the same time the patients completed a questionnaire relating to GERD (age, body mass index, alcohol intake, smoking, past history of GERD, typical or atypical symptoms of GERD and symptoms of functional dyspepsia), as well as the Symptom Checklist-90 Revision (SCL-90-R) to identify psychological symptoms. We had ob- tained consent from the patient using the questionnaire. The definition of GERD was based on the Montreal definition and Asia-Pacific consensus, and the definition of FD was found on the Rome III criteria. ${ }^{1,2,21}$

\section{Typical and Atypical Symptoms of Gastroeso- phageal Reflux Disease}

We considered heartburn and/or acid regurgitation as typical GERD symptoms. Heartburn was defined as having more than one of the following 4 symptoms more than once a week.

(1) Burning or stinging sensation of the anterior chest

(2) Burning or hot sensation of the substernal area or pit of the stomach

(3) Burning sensation like having powdered red pepper on the chest

(4) Hot and uncomfortable sensation when drinking water

We defined the symptom of regurgitation as a perception of refluxed gastric contents in the mouth or hypopharynx more than once a week. Atypical GERD symptoms including hoarseness, globus and chronic cough were also examined. Hoarseness was defined as having a horse throat, and globus as having the sensation of a foreign body in the throat or pit of the stomach. Chronic cough was defined as coughing at night or frequent coughing without having a cold. Atypical symptoms were scored when they occurred more than once a week.

Patients with typical GERD symptoms were classified as belonging to the symptomatic erosive esophagitis (SEE) group and patients without typical symptoms were classified as belonging to the asymptomatic erosive esophagitis (AEE) group. Therefore, patients without typical GERD symptoms were classified in the AEE group whether they had atypical GERD symptoms or symptoms of FD or no symptoms.

\section{Symptoms of Functional Dyspepsia}

Symptoms of FD included epigastric pain, epigastric burning, early satiation and postprandial fullness. Epigastric pain or burning was defined as painful or burning sensation in the epigastric area at least once a week during the previous 3 months, with onset at least 6 months previously, and this symptom had to be discontinuous and not relieved by defecation. Early satiation was defined as a feeling that the stomach was overfull soon after starting to eat so that the meal could not be finished. This symptom should have occurred more than 3 times per week over the previous 3 months, with onset at least 6 months previously. Postprandial fullness was an unpleasant sensation like the pro- 
longed persistence of food in the stomach occurring after ordinary-sized meals, at least 3 times a week over the previous 3 months, with onset at least 6 months previously.

\section{Symptom Checklist-90 Revision}

SCL-90-R is a simple questionnaire of 90 items, each of which is rated on a 5-point scale of distress (0-4) ranging from "not at all" to "extremely". SCL-90-R is used to evaluate psychological symptoms in 9 symptom dimensions. We used the Korean edition of SCL-90 modified and standardized by Kim et $\mathrm{al}^{22}$ in 1984.

The 9 primary symptom dimensions are referred to: somatization, obsessive-compulsive behavior, interpersonal sensitivity, depression, anxiety, hostility, phobic anxiety, paranoid ideation and psychoticism. There are also 3 global indices: the global severity index (GSI), positive symptom distress index (PSDI) and positive symptom total (PST). GSI is the mean score for all 90 items. It is designed to measure overall psychological distress. The PSDI is designed to measure the intensity of symptoms, while the PST reports the number of self-reported symptoms.

\section{Statistical Methods}

Statistical analyses were performed using SPSS version 12.0 for Windows (SPSS Inc., Chicago, IL, USA). A $P$-value of less than 0.05 was considered to be significant in all the analyses.

\section{Results}

\section{Clinical Characteristics of the Patients}

A total of 55 patients were enrolled in the study. The SEE group included 29 patients (22 males) and the AEE group 26 patients (22 males). Mean age was 48.4 years in the SEE group, 49.4 years in the AEE group and BMI was 24.2 in the SEE group, 22.6 in the AEE group. Fifteen patients in the SEE group drank more than 2 or 3 times per week $(51.7 \%)$, and 14 (53.8\%) in the AEE group. The number of smokers was 11 $(37.9 \%)$ in the SEE group and 6 in the AEE group. There was no statistically significant difference between the 2 groups in age, body mass index (BMI), alcohol drinking or smoking status (Table).

\section{Los Angeles Classification and Previous His- tory of Gastroesophageal Reflux Disease}

Patients with erosive esophagitis LA-A or more were enrolled in this study. In the SEE group, $13(44.8 \%)$ were LA-A, 12 (41.4\%) LA-B, 3 (10.3\%) LA-C and 1 (3.4\%) LA-D. In the AEE group, 19 (73.1\%) were LA-A, 7 (26.9\%) LA-B, and none LA-C or LA-D (Fig. 1). The SEE group had more severe levels of esophagitis (LA-B or more) than the AEE group but it was not statistically significant $(P=0.055)$. Fourteen patients (48.3\%) had previous history of GERD in the SEE group and 6 $(23.1 \%)$ in the AEE group and this difference did not attain statistical significance, either $(P=0.052)$.

\section{Association With Atypical Gastroesophageal Reflux Disease Symptoms}

Twenty subjects $(68.9 \%)$ in the SEE group complained of globus, and $5(19.2 \%)$ in the AEE group $(P<0.001)$. Fifteen (51.7\%) complained of chronic cough in the SEE group, and 4 $(15.3 \%)$ in the $\operatorname{AEE}$ group $(P=0.009)$. There was no difference in terms of hoarseness between the SEE and AEE groups (13 (44.8\%) vs 6 (23.0\%), $P=0.090$, Fig. 2).

\section{Association With Symptoms of Functional Dy- spepsia}

There were $11(37.9 \%), 11(37.9 \%)$ and 17 (58.6\%) patients with epigastric pain, early satiation, and postprandial fullness, re-

Table. Baseline Characteristics of Patients

\begin{tabular}{lccc}
\hline & $\begin{array}{c}\text { Symptomatic erosive } \\
\text { esophagitis }(\mathrm{n}=29)\end{array}$ & $\begin{array}{c}\text { Asymptomatic erosive } \\
\text { esophagitis (n=26) }\end{array}$ & $P$-value \\
\hline M:F & $22: 7$ & $22: 4$ & \\
Age (mean $\pm \mathrm{SD}, \mathrm{yr})$ & $48.4 \pm 13.3$ & $49.4 \pm 12.6$ & $P=0.791$ \\
BMI (mean $\pm \mathrm{SD})$ & $24.2 \pm 2.8$ & $22.6 \pm 5.2$ & $P=0.166$ \\
Frequent alcohol consumption $(>$ 2-3/wk) (n [\%]) & $15(51.7)$ & $14(53.8)$ & \\
Smoking (n [\%]) & $11(37.9)$ & $6(23.1)$ & $P=0.494$ \\
\hline
\end{tabular}

$\mathrm{M}$, male; F, female; BMI, body mass index.

Frequent alcohol consumption: more than twice a week. 




Symptomatic erosive esophagitis

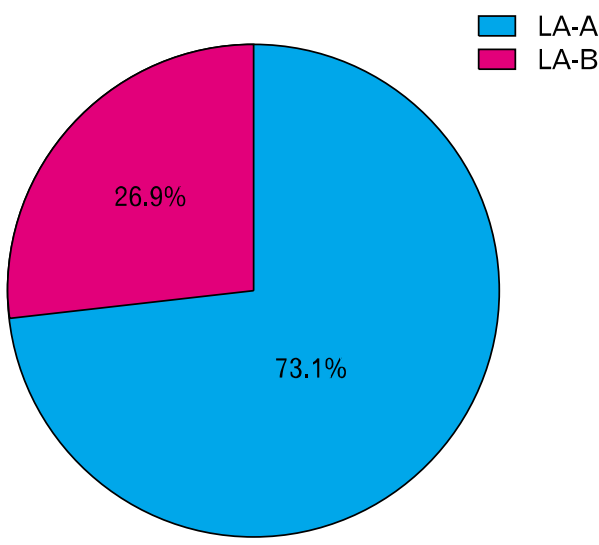

Asymptomatic erosive esophagitis
Figure 1. Los Angeles (LA) classification in the symptomatic erosive esophagitis group, 13 (44.8\%) were LA-A, $12(41.4 \%)$ LA-B, 3 (10.3\%) LA-C and $1(3.4 \%)$ LA-D. In the asymptomatic erosive esophagitis group, 19 (73.1\%) were LA-A, 7 (26.9\%) LA-B, and none LA-C or LA-D.

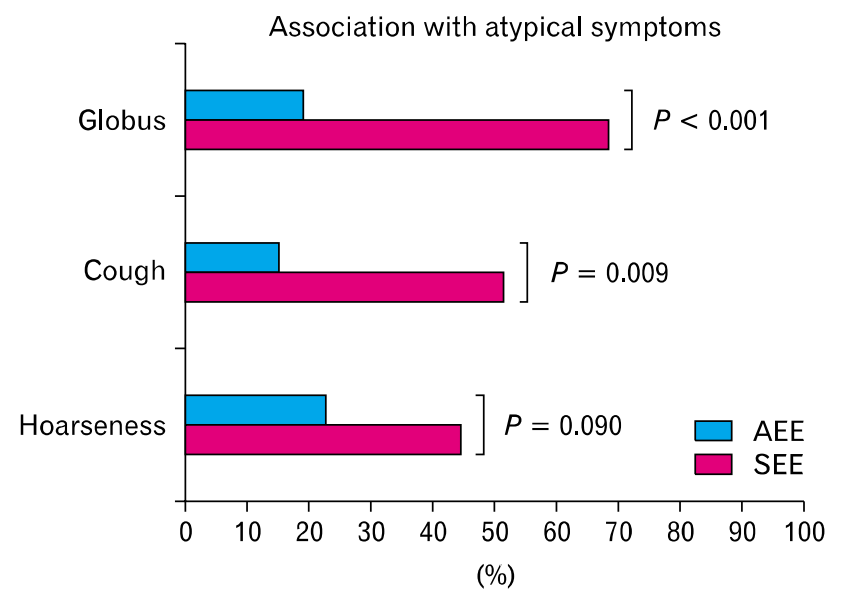

Figure 2. Association with atypical symptoms. Twenty subjects $(68.9 \%)$ in the symptomatic erosive esophagitis (SEE) group complained of globus, and $5(19.2 \%)$ in the asymptomatic erosive esophagitis (AEE) group $(P<0.001)$. Fifteen $(51.7 \%)$ complained of chronic cough in the SEE group, and $4(15.3 \%)$ in the AEE group $(P$ $=0.009$ ).

spectively, in the SEE group, and 1 (3.8\%), 1 (3.8\%) and 5 (19.2\%), respectively, in the AEE group. The SEE group outnumbered the AEE group in all symptoms and this difference was statistically significant (epigastric pain, $P=0.003$; early satiation, $P=0.003$; postprandial fullness, $P=0.005$ ) (Fig. 3 ).

\section{Association With Psychological Symptoms Using Symptom Checklist-90 Revision}

Fifty-three of the 55 patients filled out SCL-90-R; 27 in the SEE group and 26 in the AEE group. In the SEE and AEE groups, the mean values for somatization, obsessive-compulsive behavior, interpersonal sensitivity, depression, anxiety, hostility,

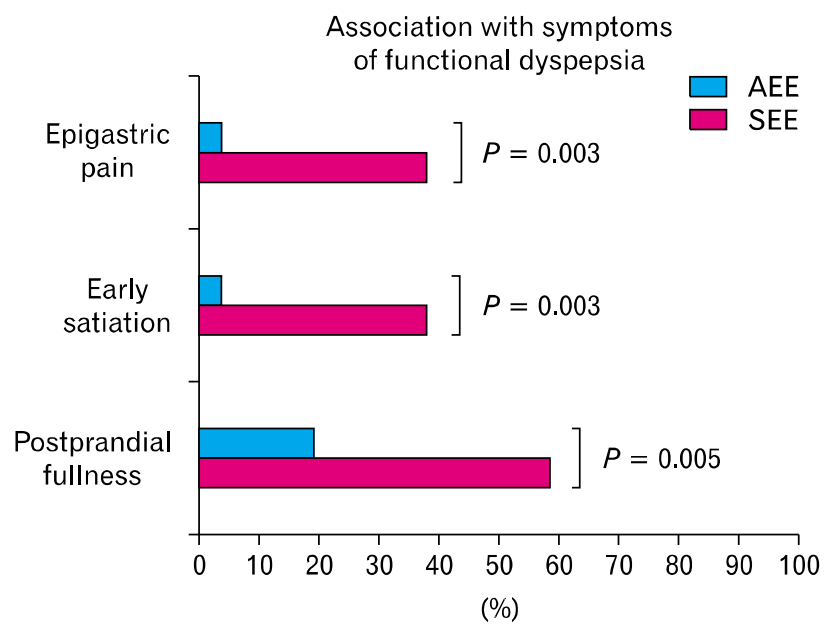

Figure 3. Association with symptoms of functional dyspepsia. There were $11(37.9 \%), 11(37.9 \%)$ and $17(58.6 \%)$ patients with epigastric pain, early satiation, and postprandial fullness, respectively, in the symptomatic erosive esophagitis group, and $1(3.8 \%), 1(3.8 \%)$ and 5 $(19.2 \%)$, in the asymptomatic erosive esophagitis group. This difference was statistically significant (epigastric pain, $P=0.003$; early satiation, $P$ $=0.003$; postprandial fullness, $P=0.005$ ). SEE, symptomatic erosive esophagitis; AEE, asymptomatic erosive esophagitis.

phobic anxiety, paranoid ideation and psychoticism were (SEE/ AEE) 52.37/44.62, 46.22/41.50, 45.74/44.77, 44.63/42.81, $45.89 / 43.12,45.56 / 44.23,49.59 / 44.65,46.48 / 43.35$ and $46.00 /$ 43.92 , respectively. The scores in all categories were higher in the SEE group than the AEE group, but only the differences for somatization $(P=0.004)$, obsessive-compulsive behavior $(P=$ $0.041)$, and phobic anxiety $(P=0.022)$ were statistically significant (Fig. 4). The GSI, PSDI and PST were 46.30/41.96, 48.81/40.04 and 45.81/42.12 in the SEE and AEE groups, 

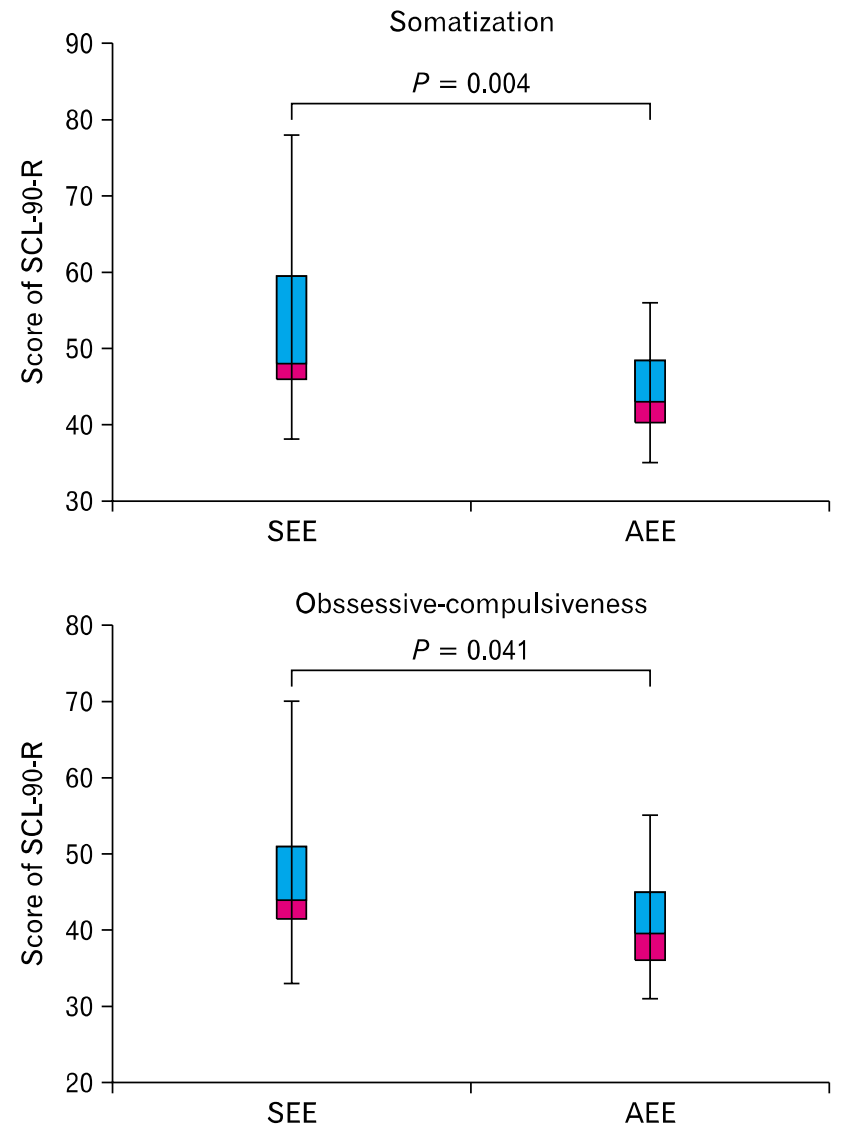

respectively. Only the difference in PSDI was statistically significant (GSI, $P=0.063$; PSDI, $P=0.009$; PST, $P=0.224$ ).

\section{Discussion}

Complications due to reflux, and lower quality of life because of reflux symptoms, are generally included in gastroesophageal reflux disease (GERD). ${ }^{23}$ Reflux symptoms refer to typical reflux related symptoms (heartburn and acid regurgitation) and atypical symptoms (chest pain, dysphagia, globus, indigestion, cough, asthma, bronchitis, pneumonia and hoarseness etc). Among patients with GERD, those with endoscopically confirmed-erosion are classified as erosive esophagitis. In most studies, about a third of patients with erosive esophagitis had no symptoms, and only a quarter of patients with reflux symptom had erosive esophagitis. ${ }^{24}$ Asymptomatic erosive esophagitis is often discovered during upper gastric endoscopy, and several studies have reported these types of GERD as asymptomatic GERD or silent GERD. ${ }^{25}$

We investigated the relationship between the presence of typical symptoms and psychological factors, as well as the clinical

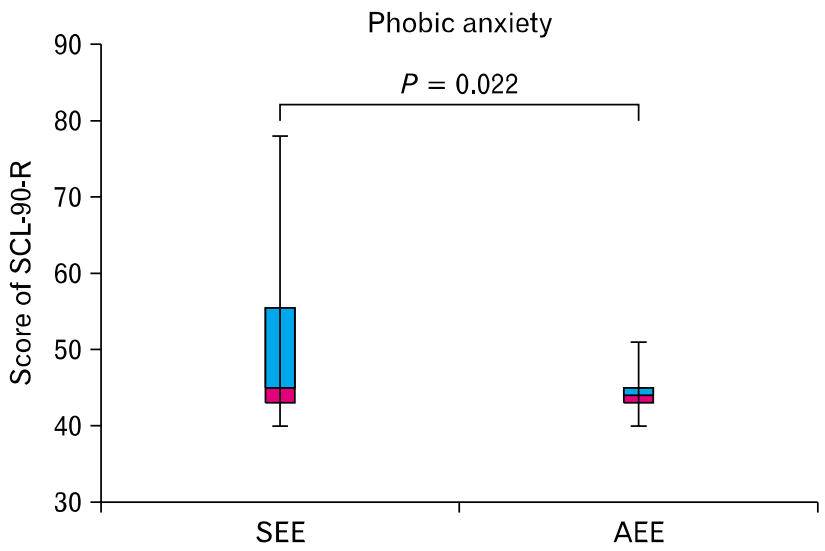

Figure 4. Association with psychiatric symptoms. The mean values for somatization, obsessive-compulsive behavior and phobic anxiety were (symptomatic erosive esophagitis/asymptomatic erosive esophagitis) $52.37 / 44.62,46.22 / 41.50$ and $49.59 / 44.65$, respectively. Somatization $(P=0.004)$, obsessive-compulsive behavior $(P=0.041)$ and phobic anxiety $(P=0.022)$ were statistically significant. SEE, symptomatic erosive esophagitis; AEE, asymptomatic erosive esophagitis; SCL-90R, Symptom Checklist-90 Revision.

characteristics of patients with erosive esophagitis. Several similar papers have been published previously. ${ }^{15,16,18,19}$ However, most of them failed to apply strict criteria for esophagitis or for the various symptoms. In this study, we set strict criteria for defining subjects' symptoms and for classifying erosive esophagitis. Our study was conducted with patients diagnosed with erosive esophagitis LA-A to D based on endoscopy. Subjects with minimal changes in the Z-line were excluded because of the uncertainty over its diagnostic interpretation. Because it is difficult to prove the association of atypical GERD symptoms with GERD, only subjects with typical GERD symptoms defined as SEE group. As in many other studies, we enrolled only patients experiencing typical GERD symptoms more than once a week.

Old age, male sex, race, family history, higher socioeconomic status, higher BMI level and smoking, are well known risk factors for typical GERD. However, the risk factors for asymptomatic GERD remain unclear. Age, BMI, alcohol intake, smoking and gender were investigated in the present study, and those risk factors did not differ between the SEE and AEE groups. However, Cho et $\mathrm{al}^{20}$ have claimed that AEE was more strongly 
associated with old age and male sex than SEE, and Nozu and Komiyama $^{26}$ stated that smoking, male gender, and lower BMI wereindependent factors associated with AEE. A larger study is needed because numbers of patients in the studies performed so far, including our own, have been small.

According to earlier studies, GERD symptoms do not predict the severity of esophagitis. However in our study which only considered erosive esophagitis, we found that patients with typical symptoms of GERD had more severe levels of esophagitis, of LA-B or more, even though there was no statistical significance. In particular, all the patients with LA-C and D esophagitis had typical symptoms. Therefore when severe esophagitis classified as LA-C or more is confirmed by endoscopy, we may assume that it is probably accompanied by typical symptoms.

In this study, patients with typical symptoms were more likely to also have atypical symptoms and symptoms of functional dyspepsia, compared to patients without typical symptoms. Many previous studies have led to the same conclusion. ${ }^{27,28}$ There is an overlap between reflux symptoms, irritable bowel syndrome, and functional dyspepsia, and IBS and FD are common over the entire spectrum of GERD. ${ }^{27}$ Rey et $\mathrm{al}^{28}$ found that the atypical symptoms of GERD were closely correlated with the typical symptoms.

Psychological disorders have been associated with various gastrointestinal diseases including GERD. ${ }^{29}$ Bile acid secretion and gastric motility are known to be affected by the emotion and stress. ${ }^{30}$ McDonald-Haile et al ${ }^{19}$ reported that relaxation training can improve symptoms of reflux and esophageal acid exposure. Baker et $\mathrm{al}^{18}$ researched 51 patients with GERD and 43 control subjects, and they suggested that depression, somatization, anxiety and intensity of reporting symptom distress were more common in GERD patients. Johnston et $\mathrm{al}^{31}$ reported that phobia, obsessionality and somatization disorder were more common in people who visited hospital with sensation of heartburn. A study by Núñez-Rodríguez and Miranda Sivelo ${ }^{15}$ also showed that patients with reflux symptom scored higher than the controls in somatization, obsessiveness, interpersonal sensitivity, phobia, psychosis and Global Index. Kamolz et $\mathrm{al}^{32}$ and Biertho et $\mathrm{al}^{33}$ found that postoperative outcomes of anti-reflux surgery of patients with psychological disorder were worse than those of a control group. These results suggest that psychological factors can influence the perception and/or severity of GERD. We therefore investigated the correlation between psychological factors and the presence of typical GERD symptoms in patients with erosive esophagitis. The scores of patients with symptomatic erosive esophagitis were higher than those of asymptomatic patients on all items of SCL-90-R, and somatization, obsessive-compulsive behavior, phobic anxiety and PSDI were statistically significant.

This study had several limitations. The first was the small sample size. Hence, a well-designed multicenter study is needed to obtain a statistically significant result. The second limitation is derived from the difficulties that the study subjects had in understanding the contents of questionnaire. Lay persons were not able to understand the specific symptoms of esophagitis and functional dyspepsia, and the psychological examination consisted of too many question lists to answer, so that 2 subjects gave up doing the psychological test. The third limitation of the study was that even if a patient reported symptoms of functional dyspepsia he was not diagnosed with functional dyspepsia because symptoms of functional dyspepsia could also be reported in erosive esophagitis. The Rome III criteria of functional dyspepsia require one or more of the following symptoms: bothersome postprandial fullness, early satiation, epigastric pain, epigastric burning with no evidence of structural disease even with, the use of endoscopy. These criteria have to be fulfilled for at least 3 months with symptom onset at least 6 months earlier. ${ }^{21}$ In this study, endoscopy was performed on all the patients and all were diagnosed with structural disease or "erosive esophagitis" by endoscopy. Therefore they could not be diagnosed with functional dyspepsia. Fourth, some of the patients with atypical reflux symptoms were classified in the asymptomatic erosive esophagitis group because only the patients with typical symptoms were included in the symptomatic erosive esophagitis group. Therefore, the lack of agreement with previous studies on the relationship between reflux symptoms, clinical characteristics and GERD severity may be attributable to differences in the classification of patients. Fifth, we did not evaluate the severity of symptoms such as frequency and strength. Therefore we might have ignored the correlation between the severity of symptom and the presence of esophagitis.

In conclusion, this study shows that reflux symptoms in patients with endoscopically-demonstrated esophagitis are associated with psychosocial factors. In addition, reflux symptoms are associated with atypical symptoms of GERD and functional dyspepsia. These findings suggest that in managing GERD patients we should investigate their psychological status and include caring for their stressors and that we should assess dyspeptic symptoms as well as the atypical GERD symptoms that often occur along with GERD symptoms. We suggest that it would be helpful in the management of GERD patients unresponsive to normal treatment to assess whether they have psychological disorders or other dyspeptic symptoms. 


\section{References}

1. Fock KM, Talley NJ, Fass R, et al. Asia-Pacific consensus on the management of gastroesophageal reflux disease: update. J Gastroenterol Hepatol 2008;23:8-22.

2. Vakil N, van Zanten SV, Kahrilas P, Dent J, Jones R; Global Consensus Group. The Montreal definition and classification of gastroesophageal reflux disease: a global evidence-based consensus. Am J Gastroenterol 2006;101:1900-1920.

3. Locke GR 3rd, Talley NJ, Fett SL, Zinsmeister AR, Melton LJ 3rd. Prevalence and clinical spectrum of gastroesophageal reflux: a population-based study in Olmsted County, Minnesota. Gastroenterology 1997;112:1448-1456.

4. Spechler SJ. Epidemiology and natural history of gastro-oesophageal reflux disease. Digestion 1992;51(suppl 1):24-29.

5. Zagari RM, Fuccio L, Wallander MA, et al. Gastro-oesophageal reflux symptoms, oesophagitis and Barrett's oesophagus in the general population: the Loiano-Monghidoro study. Gut 2008;57:1354-1359.

6. Nebel OT, Fornes MF, Castell DO. Symptomatic gastroesophageal reflux: incidence and precipitating factors. Am J Dig Dis 1976;21: 953-956.

7. Cho YS, Choi MG, Jeong JJ, et al. Prevalence and clinical spectrum of gastroesophageal reflux: a population-based study in Asan-si, Korea. Am J Gastroenterol 2005;100:747-753.

8. Oh JH, Choi MG, Kim HR, et al. [Clinical spectrum of endoscopic reflux esophagitis in routine check-up subjects in Korea.] Korean J Neurogastroenterol Motil 2006;12:12-18. [Korean]

9. Yang SY, Lee OY, Bak YT, et al. Prevalence of gastroesophageal reflux disease symptoms and uninvestigated dyspepsia in Korea: a population-based study. Dig Dis Sci 2008;53:188-193.

10. Jeon SG, Sohn CI, Kim JE, et al. [Prevalence of gastroesophageal reflux in routine check-up subjects.] Korean J Med 2000;58:145-151. [Korean]

11. Yoo SS, Lee WH, Ha J, et al. [The prevalence of esophageal disorders in the subjects examined for health screening.] Korean $\mathrm{J}$ Gastroenterol 2007;50:306-312. [Korean]

12. Hwang JK, Kim J, Hong SG, et al. [A prospective multicenter study on the prevalence and symptoms of erosive reflux esophagitis in secondary and tertiary hospitals in Korea.] Korean J Gastroenterol 2009;53:283-291. [Korean]

13. Youn YH, Kang YW, Ahn SH, Park SK. [Prevalence alteration of reflux esophagitis in recent years.] Korean J Gastrointest Endosc 2001;23:144-148. [Korean]

14. El-Serag HB. Time trends of gastroesophageal reflux disease: a systematic review. Clin Gastroenterol Hepatol 2007;5:17-26.

15. Núñez-Rodríguez MH, Miranda Sivelo A. Psychological factors in gastroesophageal reflux disease measured by scl-90-R questionnaire. Dig Dis Sci 2008;53:3071-3075.

16. Oh JH, Kim TS, Choi MG, et al. Relationship between psychological factors and quality of life in subtypes of gastroesophageal reflux disease. Gut Liver 2009;3:259-265.

17. Lee YC, Wang HP, Chiu HM, et al. Comparative analysis between psychological and endoscopic profiles in patients with gastroeso- phageal reflux disease: a prospective study based on screening endoscopy. J Gastroenterol Hepatol 2006;21:798-804.

18. Baker LH, Lieberman D, Oehlke M. Psychological distress in patients with gastroesophageal reflux disease. Am J Gastroenterol 1995; 90:1797-1803.

19. McDonald-Haile J, Bradley LA, Bailey MA, Schan CA, Richter JE. Relaxation training reduces symptom reports and acid exposure in patients with gastroesophageal reflux disease. Gastroenterology 1994;107:61-69.

20. Cho JH, Kim HM, Ko GJ, et al. Old age and male sex are associated with increased risk of asymptomatic erosive esophagitis: analysis of data from local health examinations by the Korean National Health Insurance Corporation. J Gastroenterol Hepatol 2011;26:1034-1038.

21. Brun R, Kuo B. Functional dyspepsia. Therap Adv Gastroenterol 2010;3:145-164.

22. Kim K, Kim J, Won H. Korean manual of Symptom Checklist-90Revision. Seoul: Choong Ang Aptitude Publishing Co. 1984.

23. Kahrilas PJ, Shaheen NJ, Vaezi MF; American Gastroenterological Association Institute; Clinical Practice and Quality Management Committee. American Gastroenterological Association Institute technical review on the management of gastroesophageal reflux disease. Gastroenterology 2008;135:1392-1413.e1-e5.

24. Ronkainen J, Aro P, Storskrubb T, et al. High prevalence of gastroesophageal reflux symptoms and esophagitis with or without symptoms in the general adult Swedish population: a Kalixanda study report. Scand J Gastroenterol 2005;40:275-285.

25. Fass R, Dickman R. Clinical consequences of silent gastroesophageal reflux disease. Curr Gastroenterol Rep 2006;8:195-201.

26. Nozu T, Komiyama $\mathrm{H}$. Clinical characteristics of asymptomatic esophagitis. J Gastroenterol 2008;43:27-31.

27. Neumann H, Monkemuller K, Kandulski A, Malfertheiner P. Dyspepsia and IBS symptoms in patients with NERD, ERD and Barrett's esophagus. Dig Dis 2008;26:243-247.

28. Rey E, Elola-Olaso CM, Rodríguez-Artalejo F, Locke GR 3rd, Díaz-Rubio M. Prevalence of atypical symptoms and their association with typical symptoms of gastroesophageal reflux in Spain. Eur J Gastroenterol Hepatol 2006;18:969-975.

29. Rey E, Garcia-Alonso M, Moreno-Ortega M, Almansa C, AlvarezSanchez A, Diaz-Rubio M. Influence of psychological distress on characteristics of symptoms in patients with GERD: the role of IBS comorbidity. Dig Dis Sci 2009;54:321-327.

30. Feldman M, Walker P, Goldschmiedt M, Cannon D. Role of affect and personality in gastric acid secretion and serum gastrin concentration. Comparative studies in normal men and in male duodenal ulcer patients. Gastroenterology 1992;102:175-180.

31. Johnston BT, Gunning J, Lewis SA. Health care seeking by heartburn sufferers is associated with psychosocial factors. Am J Gastroenterol 1996;91:2500-2504.

32. Kamolz T, Bammer T, Granderath FA, Pointner R. Laparoscopic antireflux surgery in gastro-oesophageal reflux disease patients with concomitant anxiety disorders. Dig Liver Dis 2001;33:659-664.

33. Biertho L, Sanjeev D, Sebajang H, Antony M, Anvari M. The influence of psychological factors on the outcomes of laparoscopic Nissen fundoplication. Ann Surg Innov Res 2007;1:2. 